\title{
THERAPY EFFECTS AND SAFETY OF RHODIOLA ROSEA EXTRACT WS 1375 IN SUBJECTS WITH SYMPTOMS OF CHRONIC FATIGUE
}

\author{
Lekomtseva Y. ${ }^{1}$, Zhukova I. ${ }^{2}$, Tartakovsky I. ${ }^{3}$ \\ ${ }^{1}$ Institute of Neurology, Psychiatry and Narcology, Academy of Medical Science, Outpatient Department, Kharkiv, Ukraine \\ ${ }^{2}$ Siberian State Medical University, Tomsk, Russian Federation \\ ${ }^{3} \mathrm{Dr}$. Willmar Schwabe GmbH \& Co. KG, Karlsruhe, Germany
}

Rhodiola rosea L. or "Golden Root" extract is characterized in the current monograph of the Committee on Herbal Medicinal Products reflection paper as a traditional herbal medicinal product for relief of symptoms of stress such as fatigue and sensation of weakness [EMEA/HMPC/232091/2011, 27 March 2012]. Clinical studies conducted with Rhodiola rosea revealed improvement in depression symptoms, mental and physical work performance, elimination of stress related fatigue and asthenia. In general, extract from Rhodiola rosea is considered to be one of the most effective adaptogenic herbal medications. Rhodiola rosea extract $\mathrm{WS}^{\circledR} 1375^{1}$ demonstrated clinical effectiveness in improvement of life-stress symptoms (Edwards, 2012).Unexplained chronicfatigue (CF) is a widespread healthcare problem that significantly affects the professionally active part of population. In this regard it is expected that extract WS ${ }^{\circledR} 1375$ may significantly improve well-being of these patients, where lifestress often acts as a trigger factor of CF.

The objective of this open-label multicenter study was the evaluation of therapeutic effects and safety of Rhodiola rosea extract WS ${ }^{\circledR} 1375$ in subjects with symptoms of CF. Treatment effects were measured on $\mathrm{CF}$ symptoms, quality of sleep, concentration, post exertional malaise, and level of daily activity.

Study design:an open-label multicenter, single arm study conducted in Ukraine.

Subjects:100 male and female outpatients (aged 18 to 60 years) with symptoms of CF.

Specific Inclusion Criteria:

- Clinically evaluated, unexplained persistent or relapsing fatigue symptoms lasted at least 2 months that are not the result of ongoing exertion, are not substantially relieved by rest, results in substantial reduction in previ-

\footnotetext{
${ }^{1}$ WS ${ }^{1} 1375$ is the active substance of Vitano ${ }^{\circledR}$ (Dr. Willmar Schwabe
} GmbH \& Co. KG, Karlsruhe, Germany). ous levels of occupational, educational, social or personal activities.

- The perceived symptoms of CF listed below assessed as $\geq 5$ on Numerical Analogue Scales (NAS): postexertional malaise (extreme prolonged exhaustion following physical or mental exertion) lasting more than 24 hours, substantial impairment in short-time memory and concentration, unrefreshing sleep.

- Multidimensional Fatigue Inventory 20 (MFI-20) score 7 or more for the sub-scales: general fatigue, physical fatigue, mental fatigue.

Treatment: $200 \mathrm{mg}$ of Rhodiola rosea extract $\mathrm{WS}^{\circledR}$ 1375 twice daily; duration of treatment was eight weeks with visits at Screening (Day -2) Baseline (Day 0), Week 1, Week 4, and Week 8.

Outcome Variables:MFI-20 - assessment of the chronic fatigue symptoms, 3 Numerical Analogue Scales of $\mathrm{CF}$ symptoms (post-exertional malaise, impaired memory and concentration; unrefreshing sleep), Sheehan Disability Scale (SDS) - assessment of the impairment of daily living and reduction in previous levels of activity, Number Connecting Test (NCT) - assessment of memory and concentration, Pittsburgh Sleep Quality Index (PSQI) - assessment of sleep quality, Recent Perceived Stress Questionnaire (PSQ-R) - assessment of stress level,Beck's Depression Inventory (BDI) - assessment of depression, Clinical Global Impression (CGI).

From 101 patients with symptoms of CF who started the study treatment the data of 100 was included in IntentTo-Treat (FAS) analyses.

Statistically significant improvement $(p<0.001)$ was observed for all outcome variables. Subjects showed improvement not only in specific $\mathrm{CF}$ outcomes, but also in concomitant conditions related to $\mathrm{CF}$ comorbidity and general well-being: PSQI, PSQ, BDI-II, SDS, and NCT.

Outcome variables NAS andMFI-20 that specifically reflect symptoms of $\mathrm{CF}$ demonstrate clear improvement particularly at the first treatment week. 
Adverse events reported 41 study participants. All events were connected with concomitant conditions and estimated as unrelated or unlikely related to Rhodiola rosea extract WS ${ }^{\circledR} 1375$.

Effectiveness of Rhodiola rosea extract WS ${ }^{\circledR} 1375$ in the treatment of CF correlates with previous publications where strong adaptogenic and anti-stress effects of Rhodiola rosea were demonstrated (Panossian, 2010; Olsson, 2009). Improvement of CF symptoms and general well-being correlates with reduction of patient's life-stress level. This hints at a potential causal relationshipbetween life-stress and CF symptoms.

Safety and tolerability of WS ${ }^{\circledR} 1375$ also presented a favorable profile.

Conclusion: Rhodiola rosea extract $\mathrm{WS}^{\circledR} 1375$ at a dose of $200 \mathrm{mg}$ twice daily is effective and safe in sub- jects with unexplained CF symptoms and concomitant conditions, and may be helpful in everyday clinical practice of general practitioners and psychoneurologists.

\section{References}

1.Edwards D., Heufelder A. and Zimmermann A. Therapeutic Effects and Safety of Rhodiola rosea Extract WS 1375 in Subjects with Life-stress Symptoms - Results of an Openlabel Study, Phytother. Res. (2012), Wiley Online Library (wileyonlinelibrary.com) DOI: 10.1002/ptr.3712.

2. EMEA/HMPC/232091/2011: Community herbal monograph on Rhodiola rosea L., rhizomaet radix, Final, London, 27 March 2012.

3. Panossian A., Wikman G., Sarris J. Rosenroot (Rhodiola rosea): Traditional use, chemical composition, pharmacology and clinical efficacy, Phytomedicine 17 (2010) 481-493.

4. Olsson E., von Schéele B., Panossian A. A Randomised, Double-Blind, Placebo-Controlled, Parallel-Group Study of the Standardised Extract SHR-5 of the Roots of Rhodiola rosea in the Treatment of Subjects with Stress-Related Fatigue, Planta Med 2009; 75(2): 105-112.

Acknowledgments: This work was supported by grants from Dr. Willmar Schwabe Pharmaceuticals. We would like to thank the clinical investigators who enrolled and cared for study patients: Dr. Peleshok S, Prof. Kozelkin A, Prof, Litovchenko T, Prof. Litvinenko N., Prof. Voloshin, Dr. Medvedkova S., Dr. Neryanova Y., Dr. Kuznetsov A., Dr. Pasiura I., Dr. Zaval'na O., Dr. Tondii O., Dr. Del'va M., Dr, Del'va I.

\title{
ЭФФЕКТИВНОСТЬ И БЕЗОПАСНОСТЬ ЛЕЧЕНИЯ ЭКСТРАКТОМ РОДИОЛЫ РОЗОВОЙ WS 1375 ПАЦИЕНТОВ С СИНАРОМОМ ХРОНИЧЕСКОЙ УСТАЛОСТИ
}

\author{
Лекомцева Е.В. ${ }^{1}$ Жуккова И.А. ${ }^{2}$, Тартаковский И.А. ${ }^{3}$ \\ ${ }^{1}$ Институт неврологии, психиатрии и наркологии, Академия медицинских наук, г. Харьков, Украина \\ ${ }^{2}$ Сибирский государственный медицинский университет, г. Томск, Россия \\ ${ }^{3}$ Dr. Willmar Schwabe GmbH \& Co. KG, г. Карлсруе, Германия
}

\begin{abstract}
Экстракт родиолы розовой, или золотой корень, описан в монографии Комитета по фитотерапии как традиционное травяное лекарственное средство для облегчения симптомов стресса, таких как усталость и ощущение слабости [2]. Клинические исследования, проведенные с родиолой розовой, показали снижение проявлений симптомов депрессии, повышение физической работоспособности, устранения усталости и астении, связанных со стрессом. Экстракт родиолы розовой считается одним из наиболее эффективных адаптогенов растительного происхождения. Экстракт родиолы розовой WS 1375 продемонстрировал клиническую эффективность в снижении проявлений симптомов стресса [1] (Edwards, 2012). Необъяснимая хроническая усталость является широко распространенной проблемой здравоохранения, которая оказывает существенное влияние на профессионально активную часть населения. В связи с этим ожидается, что
\end{abstract}

экстракт WS 1375 может значительно улучшить состояние пациентов, у которых повседневный стресс часто выступает в качестве триггерного фактора хронической усталости.

Цель данного исследования - оценка эффективности и безопасности лечения экстрактом родиолы розовой WS 1375 пациентов с синдромом хронической усталости. Эффект лечения оценивали по симптомам хронической усталости, качеству сна, концентрации, физической нагрузке, и уровню повседневной активности.

Дизайн исследования - открытое многоцентровое исследование, проводимое в Украине.

Обследованы 100 мужчин и женщин амбулаторного наблюдения (в возрасте от 18 до 60 лет) с симптомами хронической усталости.

Критерии включения в исследование: клинические значимые, необъяснимые постоянные или рецидивирующие симптомы усталости, длящиеся не менее 2 мес, 
которые не являются результатом существующей нагрузки, не проходящие после отдыха, приводящие к существенному снижению уровня профессиональной, познавательной, социальной или личной активности.

Восприятие симптомов хронической усталости оценивалось как $\geq 5$ по визуальной аналоговой шкале (ВАШ): общее недомогание (длительное истощение после физического или умственного напряжения) продолжительностью более 24 ч, существенное влияющее на кратковременную память и концентрацию внимания, качества сна.

Использовался многомерный опросник для оценки астении (MFI-20): оценивались общая усталость, физическая усталость, умственная усталость.

Лечение: 200 мг экстракта родиолы розовой WS 1375 два раза в день, продолжительность лечения составила 8 нед с визита скрининга (день 2) исходный (день 0), 1-я неделя, 4-я неделя и 8-я неделя.

Оценивающие параметры: MFI-20 - оценка симптомов хронической усталости; три визуальноаналоговые шкалы для оценки симптомов хронической усталости (общее недомогание, нарушения памяти и концентрации внимания, снижение качества сна); шкала нетрудоспособности Шихана (SDS) - оценка влияния на повседневную активность и уменьшение относительно предыдущего уровня; количественный тест переключения (NCT) - оценка памяти и концентрации внимания, Питсбургская шкала качества сна (PSQI) - оценка качества сна; анкета восприятия стресса (PSQ-R) - оценка уровня стресса; шкала депрессии Бека (BDI) - оценка депрессии; шкала общего клинического впечатления (CGI).

Статистически значимое улучшение $(p<0,001)$ наблюдалось для всех изучаемых переменных. Пациенты показали улучшение не только в конкретных результатах хронической усталости, но и в других симптомах, связанных с сопутствующими хронической усталости заболеваниями и общего улучшения: PSQI, PSQ, BDI-II, SDS и NCT.

Изучаемые показатели ВАШ и MFI-20, которые специфически отражали симптомы хронической уста- лости, показали явное улучшение особенно на первой неделе лечения.

Побочные явления были обнаружены у 41 участника исследования. Все побочные явления были связаны с сопутствующими заболеваниями и оценивались как не связанные или маловероятно связанные с применением экстракта родиолы розовой WS 1375.

Эффективность применения экстракта родиолы розовой WS 1375 для лечения хронической усталости подтверждается данными и других исследователей, в которых продемонстрирован сильный адаптогенный и антистрессовый эффекты родиолы розовой (Panossian, 2010; Olsson, 2009). Снижение симптомов хронической усталости коррелирует с уменьшением уровня стресса пациентов. Это указывает на потенциальную причинноследственную связь между повседневным стрессом и симптомами хронической усталости.

Безопасность и переносимость WS 1375 также показала благоприятный профиль.

Таким образом, экстракт родиолы розовой WS 1375 в дозе 200 мг два раза в день является эффективным и безопасным у пациентов с необъяснимыми симптомами хронической усталости и сопутствующих заболеваний, и может быть рекомендован в повседневной клинической практике врачей общей практики и психоневрологов.

\section{Литература}

1.Edwards D., Heufelder A., Zimmermann A. Therapeutic Effects and Safety of Rhodiola rosea Extract WS 1375 in Subjects with Life-stress Symptoms - Results of an Open-label Study, Phytother. Res. (2012), Wiley Online Library (wileyonlinelibrary.com) DOI: 10.1002/ptr.3712.

2. EMEA/HMPC/232091/2011: Community herbal monograph on Rhodiola rosea L., rhizomaet radix, Final, London, 27 March 2012.

3.Panossian A., Wikman G., Sarris J. Rosenroot (Rhodiolarosea): Traditional use, chemical composition, pharma-

cology and clinical efficacy, Phytomedicine 17 (2010) 481493.

4. Olsson E., von Schéele B., Panossian A. A Randomised, Double-Blind, Placebo-Controlled, Parallel-Group Study of the Standardised Extract SHR-5 of the Roots of Rhodiola rosea in the Treatment of Subjects with Stress-Related Fatigue,Planta Med 2009; 75(2): 105-112.

\footnotetext{
Поступила в редакцию 26.09.2013 г.

Утверждена к печати 09.10.2013 г.
}

Благодарности: Работа была выполнена при поддержке гранта компании Dr. Willmar Schwabe Pharmaceuticals. Мы благодарны докторам, которые приняли участие в исследовании: Dr. Peleshok S., Prof. Kozelkin A., Prof, Litovchenko T., Prof. Litvinenko N., Prof. Voloshin, Dr. Medvedkova S., Dr. Neryanova Y., Dr. Kuznetsov A., Dr. Pasiura I., Dr. Zaval'na O., Dr. Tondii O., Dr. Del'va M., Dr, Del'va I.

Лекомцева Евгения Владимировна, Институт неврологии, психиатрии и наркологии, Академия медицинских наук (г. Харьков, Украина). Жукова Ирина Александровна, Сибирский государственный медицинский университет (г. Томск, Россия). 
Тартаковский Игорь Александрович, Dr. Willmar Schwabe GmbH \& Co. KG (г. Карлсруе, Германия). 